\title{
ENFOQUES CONTEMPORANEOS DEL SISTEMA COGNITIVO HUMANO: UNA REVISION CRITICA
}

Oscar Nudler

\section{Abstract}

El propósito principal de este artículo es el de describir y evaluar los supuestos centrales que subyacen a diversos enfoques psicológicos (y también filosóficos) de los procesos cognitivos humanos. Estos enfoques se dividen aquí en dos tendencias opuestas: cognitivismo y anticognitivismo. El primero se caracteriza por la aceptación de los dos siguientes supuestos: 1) Los procesos cognitivos son funcionalmente autónomos. O, dicho de otro modo, son separables de otros procesos y también entre sí. 2) Los procesos cognitivos operan de acuerdo con un conjunto de reglas que constituyen un programa, análogamente al procesamiento de la información por una computadora. Dentro del cognitivismo distinguimos, a su vez, dos variantes: el cognitivismo clásico y el cognitivismo revisado, que intenta incorporar al esquema cognitivista dimensiones de los procesos cognitivos que el cognitivismo clásico deja de lado, tales como la contextualidad y la intencionalidad. El anticognitivismo es descripto, por su parte, como la posición de los críticos más radicales del cognitivismo, es decir, de los que rechazan los dos supuestos del mismo. A lo largo de las dos primeras secciones de este artículo se presentan y examinan todas estas posiciones. Se concluye que las críticas al segundo supuesto del cognitivismo parecen acertadas pero no así, o al menos no totalmente así, las críticas al primer supuesto. A fin de presentar esta conclusión con mayor claridad, se introduce en la sección III un modelo centro-periferia del sistema cognitivo humano. A la luz de este modelo se señala que en un sistema cognitivo humano desarrollado existen procesos cognitivos que, aunque sea parcialmente, funcionan de un modo autónomo respecto de otras partes del sistema, en especial de su parte central. Esto implica que, en una versión más débil, de alcance no universal, el primer supuesto del cognitivismo retendría su validez. Pero al mismo tiempo también se reconoce que existen 
procesos, como es en general el caso de los procesos de creación, cuya comprensión requiere abandonar el supuesto cognitivista de la autonomía o compartimentalización de los procesos cognitivos y apelar a la unidad profunda del sistema cognitivo humano.

\section{El enfoque cognitivista: Supuestos basicos}

Hacia fines de la década del 50 la psicología cognitiva empezó a adquirir impulso hasta que alcanzó, unos años más tarde, una posición de predominio en la psicología académica, especialmente en los Estados Unidos. Paralelamente, se desarrollaron de un modo creciente investigaciones en el campo de la inteligencia artificial. Anbos movimientos - psicología cognitiva e inteligencia artificial - son expresiones, a nuestro juicio, de un mismo paradigma básico que denominaremos "cognitivismo" En lo que sigue daremos una breve descripción de este paradigma.

Un supuesto central del cognitivismo es la creencia en que de algún modo existe funcionando en el ser humano un "aparato cognitivo" Esto significa que el conjunto de los procesos cognitivos - formar y hacer uso de categorías, resolver problemas, percibir, recordar, imaginar, etc. - es considerado como constituyendo un sistema funcionalmente autónomo. Este supuesto de autonomía funcional no implica, por cierto, negar la inflüencia que otros procesos, tales como los llamados "procesos dinámicos" o, simplesmente, los cambios fisiológicos del organismo, pueden ejercer sobre los procesos cognitivos. Esta influencia, sin embargo, es considerada como solamente capaz de inhibir o acelerar los procesos cognitivos 0 , inclusive, de poner en marcha un proceso cognitivo en lugar de otro pero nunca como capaz de alterar la naturaleza misma de cada proceso cognitivo o de diluir la diferencia entre lo cognitivo y lo no cognitivo.

Entendido del modo que acabamos de señalar, el supuesto de autonomía funcional del sistema cognitivo humano (SCH) ha sido predominante en la filosofía de la mente y la psicología occidental desde Platón hasta Piaget. La moderna psicología cognitiva y la investigación sobre la inteligencia artificial han adoptado, como señalamos arriba, este supuesto y en este sentido pertenecen a la vieja tradición cognitivista. Sin embargo, podría arguirse que han avanzado más allá de esta tradición al tomar a la computadora como una "analogíaraíz" y considerar a los mecanismos cognitivos humanos como mecanismos de procesamiento de la información. Dice al respecto Ulrich Neisser en un libro que tanto ha contribuído al éxito de la psicología cognitiva en la comunidad académica: "Aunque la medición de la información puede ser de escaso valor para el psicólogo cognitivo, otra rama de las ciencias de la información - la programación com- 
putacional - tiene mucho más para of recerle. Un programa no es un artefacto para medir información sino una receta para seleccionarla, almacenarla, recuperarla, combinarla, emitirla y, en general, manipularla. Como lo señalan Newell, Shaw y Simon, esto significa que los programas tienen mucho en común con las teorías acerca de la cognición. Ambos son descripciones de las vicisitudes de la información recibida"

El cognitivismo contemporáneo agregaria, pues, al supuesto tradicional de la autonomía funcional del $\mathrm{SCH}$ un segundo supuesto según el cual todo proceso cognitivo es, del mismo modo que lo que ocurre con una computadora, un proceso de tratamiento de información sobre la base de un programa o un conjunto dado de reglas. Daremos a este segundo supuesto el nombre de supuesto de la inmanencia de reglas.

Bien mirado, sin embargo, este supuesto no es en realidad completamente novedoso. Para tomar unos pocos ejemplos históricos, las categorías trascendentales de Kant, la inferencia inconsciente de Helmholtz, y, más cercanamente, las estructuras de Piaget implican una similar creencia en la inmanencia de reglas en los procesos cognitivos.

Ahora bien, el supuesto de la inmanencia de reglas, común pues a las distintas formas del cognitivismo, ha tomado tradicionalmente como caso paradigmático el pensamiento abstracto, donde el uso de reglas, en particular las reglas lógicas, aparece como obvio. Por lo tanto, su aplicación a otros procesos cognitivos podría considerarse como una extensión del caso paradigmático. Intentaremos hacer más gráfica esta extensión mediante una metaforma espacial. De paso esto nos servirá para introducir una visión global del sistema cognitivo humano.

Supongamos que el cuerpo total de los conocimientos que, en un determinado momento, posee un individuo está dividido en niveles de acuerdo con su posibilidad de comunicación verbal. Tendríamos así un conjunto de niveles, desde un nivel superior formado por los conocimientos más precisos, altamente comunicables en fprma verbal, hasta un nivel inferior constituido por conocimientos no verbales en absoluto, incorporados directamente a los músculos, por decirlo así, como los implicados en el caminar, el nadar, el bailar, etc. Entre los dos niveles extremos tendríamos una ancha zona intermedia formada, en orden decreciente, por conocimientos categoriales pero no perfectamente comunicables vía lenguaje, como los conocimientos que subyacen a la mayor parte del discurso ordinario, cargado de vague-

(1) - Neisser, U. (1967) Cognitive Psychology. New York, Appleton-Century-Crofts (p. 7). 
dad y ambiguedad y, siguiendo, por conocimientos escasamente categoriales o no categoriales en absoluto, como los implicados en la percepción empática de otra persona, en la captación de un "algo" indescriptible en un ambiente, etc. Adoptando la termiología de Silvano Arieti, (2) podríamos distinguir entre conocimientos conceptuales (que van desde los más a los menos comunicables verbalmente), conocimientos endoceptuales (es decir, conocimientos de algún modo presentes en la conciencia pero no categorizados) y conocimientos exoceptuales (o sea conocimientos no presentes normalmente en la conciencia y, al menos en parte, automatizados, incorporados directamente al cuerpo). No existe, por supuesto, un límite preciso entre las distintas zonas sino más bien un continuo sólo gradualmente cabiante desde un extremo al otro. Tendríamos así el siguiente diagrama de los niveles del $\mathrm{SCH}$ :

Nivel conceptual altamente comunicable

Posibilidad decreciente de comunicación verbal
Niveles

Nivel conceptual escasamente conceptuales comunicable

Nivel endoceptual

Nivel exoceptual
Niveles no conceptuales

Fig.: 1 El sistema cognitivo humano: niveles

Dado este diagrama podríamos decir, metafóricamente, que el cognitivismo mira el SCH desde arriba y, por lo tanto, que toma como paradigma para entender todo el sistema la parte extrema superior, es decir, el nivel de los conocimientos altamente comunicables. Por consiguiente, si la actividad mental correspondiente al nivel superior ha de entenderse sobre la base de la presencia de reglas inmanentes (especificamente, reglas de manipulación de símbolos) la actividad correspondiente a niveles inferiores también ha de entenderse sobre la base de algún tipo similar de reglas.

De hecho, la extensión del supuesto de la existencia de reglas inmanentes ha procurado especialmente cubrir áreas como la percepción, la memoria, el lenguaje, etc. pero ha dejado prácticamente de

(2) - Arieti, S. (1976) The Intrapsychic Self: Feeling and Cognition in Health and Mental Illness. New York, Harper \& Row. 
lado los niveles más bajos como el de las habilidades motrices. Estas habilidades aparecen, como lo han destacado Hubert Dreyfus (3) y, más recientemente, John Haugeland (4), como una de las áreas más refractarias al enfoque cognitivista. Lo que aquí nos interesa, sin embargo, es la extensión del enfoque cognitivista al dominio intermedio de nuestra figura $y$, en especial, al campo de la percepción, campo en que los cognitivistas han invertido grandes esfuerzos para demonstrar que su enfoque realmente funciona. No trataremos en este trabajo el caso del lenguaje, área imposible de abordar en el reducido espacio disponible.

\section{Cognitivismo clasico, cognitivismo revisado, anticognitivismo}

Los distintos modelos de procesamiento de la información suelen suponer, como punto de partida de su representación del proceso de la percepción, la existencia de un input sensorial aislado. A partir de aquí los modelos, aunque divergentes y hasta opuestos en los detalles, comparten una misma concepción básica de los que ocurre. Según esta concepción, el SCH aplica primero al input un conjunto de detectores de rasgos (features) de modo de obtener una lista de rasgos elementales que presenta el objeto en consideración. Esta lista constituye una descripción encodificada del objeto. $\mathrm{El} \mathrm{SCH}$ procede luego a identificar el objeto así descripto, siendo ésta la etapa de reconocimiento de pauta (pattern recognition). Esto se logra comparando el objeto construido en la etapa de detección de rasgos con prototipos almacenados en la memoria de largo plazo (LTM). En suma, como resultado final de un processo que incluye, entre otras etapas, una etapa de descomposición y análisis del input sensorial en rasgos discretos, una etapa de construcción o síntesis de una replica del objeto real y una etapa de comparación con un prototipo almacenado en la memoria de largo plazo, se obtiene un objeto percibido (o recordado, o imaginado, etc., según sean las subrutinas puestas en juego).

Esta conceptualización congnitivista que podríamos llamar clásica ha sido objeto de críticas dentro del mismo campo cognitivista. Se ha señalado que es sólo aplicable a experimentos de laboratorio en que los estímulos empleados son adrede fugaces, aislados o ambiguos de modo que el sujeto no puede incluirlos en un contexto conocido y no puede aplicar sus procedimientos habituales para enfrentarse con ellos. En un ambiente natural, en cambio, se sostiene como más apro-

(3) - Dreyfus, H. L. (1972) What Computers Can't'Do: A Critique of Artificial Reason. New York, Harper \& Row.

(4) - Haugeland, J. (1978) The Nature and Plausibility of Cognitivism, The Behavioral and Brain Sciences, 2. 
que se pierden en el horizonte y entre las cuales no pueden trazarse límites claros. James, con su delicado sentido de la complejidad, nos sugiere una especie de cuadro impresionista, muy alejado de los rasgos analíticos y discretos supuestos por los cognitivistas ¿Cuál es la pintura más aproximada a lo que "realmente ocurre"?

Supongamos un acto cognoscitivo ordinario: la percepción del árbol que está detrás de mi ventana. Un modelo de procesamiento de la información requeriría, por empezar, una distinción clara entre lo que pertence al input $\mathrm{y}$, lo que no pertenece a el. Pero icómo trazar aquí los límites del input? Yo veo el árbol pero no veo solamente el árbol. También veo, aunque con menor nitidez, la vegetación que lo rodea, la colina que está detrás, y aún más atrás, las montañas nevadas y el cielo azul. Dónde están los límites del input? ¿En los límites del árbol? Obviamente no. ¿En alguna línea imaginaria trazada en la pradera? Evidentemente tampoco. ¿En la totalidad de mi campo visual? Aún esta última posibilidad debería descartarse pues si optáramos por ella dejaríamos afuera del input elementos que forman parte de él, aunque de un modo especial: la percepción del vidrio a través del cual estoy mirando el árbol y, más allá de las percepciones visuales, la percepción de la resistencia del escritorio a la presión de mi codo apoyado en él, la percepción del crujido de la escalera de madera bajo el peso de alguien que está subiendo, la percepción cenestésica general de mi cuerpo. ¿Dónde trazar razonablemente los límites del input? La dificultad crece todavía más si pensamos que mi percepción del árbol "arrastra" con ella de un modo inextricable conocimientos que trascienden el dominio de la percepción. Yo sé, por ejemplo, que la fuente de lız que ilumina el paisaje es el sol que se encuentra a mis espaldas y cuya posición determina el largo de las sombras que percibo, sé que detrás de las montañas hay un valle y que después está el océano, etc., etc.

En mi percepción del árbol están de algún modo presentes estos conocimientos y muchos otros, imposibles de numerar exhaustivamente. Este fondo indeterminado o hallo en el sentido de James, semejante a la noción husserliana de horizonte externo, no puede ser incorporado a un programa digital de computación. Esto ya fue señalado hace varios años por Dreyfus en su crítica pionera de la inteligencia artificial: "Para una computadora, que debe tomar todo bit de información explicitamente o no tomarlo en absoluto, no puede haber horizonte externo. Toda información a tomar en cuenta debe ser tan determinada como la figura. Este horizonte externo describe cómo la información subyacente. es ignorada sin ser ex-
cluída" (9).

(9) - Dreyfus, H. Op. cit., p. 153. 
La tesis de Dreyfus fue, dicho sea de paso, implícitamente admitida por su crítico cognitivista Zenon N. Pylyshyn (10) cuando argumenta en favor de la posibilidad de un desarrollo futuro en el cual las técnicas de simulación por computadora podrían incorporar aspectos de la conciencia de halo. Esto implicaría computadoras que no trabajarían según el modelo actual de procesamiento de la información. Esto no deja de ser una posibilidad lógica pero hasta el presente no es, sin duda, un desarrollo práctico previsible sobre la base de la técnica actual.

Repitamos el final de la cita precedente: "ignorada sin ser excluída" La frase, a primera vista paradójica, apunta a poner de manifiesto el peculiar carácter del fondo que acompaña a las figuras trazadas por los actos cognitivos humanos. Se trata de un fondo presente aunque "subsidiariamente", como decía Michael Polanyi. Podría decirse que forma parte de algún modo de la figura ya que ésta no aparece como exactamente la misma en diferentes contextos. Sin embargo no forma parte de la figura del mismo modo que los elementos y las relaciones figurales. Tal vez podría afirmarse que lo hace de un modo "mas débil" aunque es difícil dilucidar el significado exacto de esto. Pero, además, no todo el contexto determina la figura del mismo modo. En primera aproximación, podría decirse que la influencia de un sector del contexto es inversamente proporcional a sua distancia de la figura. Esto, sin embargo, es un tanto sobresimplificado ya que elementos físicamente cercanos pueden ser, desde el punto de vista de un observador, psicológicamente lejanos y viceversa. Sea como fuere, es claro que parece haber en general una lenta degradación del contexto, una progresiva desaparición del mismo en dirección a límites indeterminados, y esto no es demasiado compatible con un conjunto de reglas basado en distinciones discretas.

Dejando ahora de lado el problema del contexto y yendo hacia la figura misma, o más en general, hacia lo que se sitúa en el foco de un acto cognitivo, cabe perguntarse si talvez en este caso no sería razonable sostener que la base del proceso está dada por una distinción entre rasgos discretos. Ya la Gestalttheorie había criticado una suposición de este tipo. Existen ahora bastantes evidencias de que los procesos cognitivos reales no apoyarían una respuesta positiva a la pregunta anterior. Eleanor Rosch, por ejemplo, ha revisado parte de esta evidencia en un trabajo sobre categorización en que abarca tanto la categorización de base perceptual como la de base no perceptual. Su conclusión, tanto para el caso de la percepción del color

(10) - Pylyshyn, Z.W. (1975) Minds, machines and phenomenology: Some reflections on Dreyfus' What Computers Cant't Do., Cognition, Vol. 3/1, p. 71 ss. 
como para la percepción de formas geométricas y la percepción de emociones faciales, es que la asignación de un conjunto de estímulas a una categoria no suele basarse en un proceso de análisis en términos de atributos más simples que la categoría correspondiente sino en una comparación global con un prototipo o ejemplo paradigmático de la categoría. Rosch extiende esta conclusión a todas las categorías de objetos concretos, aunque no tengan una base neurofisiológica como las anteriores: "Se han presentado elementos de prueba producidos por técnicas experimentales ampliamente convergentes que muestran que las categorías de sustantivos puedem considerarse como codificadas y usadas en términos de prototipos y distancia de prototipos" (11).

$\mathrm{Si}$ se demostrara finalmente que el $\mathrm{SCH}$ procede en general de acuerdo con este modelo, es decir, sobre la base de comparaciones y distancias con protutipos que son gestalten concretas y no listas de atributos discretos, simples y universales, la utilidad de un modelo de reglas inmanentes de procesamiento de la información quedaría por supuesto comprometida. Tal vez habría que recordar aquí que autores como Hanson, Kuhn y otros han planteado la posibilidad de un proceso no basado, o no enteramente basado, en reglas, inclusive en el caso del pensamiento altamente abstracto característico de las ciencias avanzadas, dominio generalmente considerado como un ejemplo indiscutible del modelo de procesamiento de la información. Kuhn prefiere conceptualizar al pensamiento abstracto como un proceso esencialmente similar a la percepción, entendiendo principalmente a ésta como una actividad de organización (y desorganización) de gestalten y no como una actividad de aplicación de reglas. En este sentido introduce la diferencia entre un pensamiento fundado en un paradigma y un pensamiento regido por reglas y sostiene que el primero no requiere necesariamente del segundo: " la existencia de un paradigma ni siquiera debe implicar la existencia de algún conjunto completo de reglas" (12).

Resumiendo ahora estas breves observaciones acerca de la percepción y el pensamiento, vemos que todas implican una crítica de la concepción del SCH que supone que éste funciona de acuerdo con reglas inmanentes que operan sobre la base de distinciones claras entre rasgos discretos componentes de la figura, entre la figura y su contexto y entre las partes del contexto. Se trata, pues, de un ataque

(11) - Rosch, E. Human Categorization (en prensa). En N. Warren (Ed.) Advances in Cross-cultural Psychology (Vol. 1) London, Academic Press.

(12) - Kuhn, T. (1962) The Structure of Scientific Revolutions. Chicago, University of Chicago Press, p. 44. 
al segundo supuesto del cognitivismo, el supuesto de la inmanencia de reglas. El primer principio, el principio de la autonomía funcional, permanece hasta aquí intocado. Es posible, en efecto, que el $\mathrm{SCH}$ no funciona como una computadora que procesa información de acuerdo con un programa almacenado en su memoria de largo plazo sino que funcione de algún otro modo cuya comprensión exija el uso de conceptos tales como "paradigma" y "distancia de un paradigma", "halo", "horizonte externo", "conciencia focal" vs. "conciencia subsidiaria", etc., y que, al mismo tiempo, sea funcionalmente autónomo. Pues bien, los críticos que llamaré "radicales" del cognitivismo no sólo impugnan el supuesto 2 sino también el supuesto 1 . No sólo afirman que los procesos cognitivos humanos no son procesos simulables por computadoras sino, sobre todo, que non son procesos autónomos. Ludwig Wittgenstein ha expuesto claramente una posición de este tipo: "Proveer fundamentos, justificar la evidencia, llega a un fin pero el fin no está constituído por ciertas proposiciones que se nos aparecen como inmediatamente verdaderas, es decir, no está en nuestro ver; es nuestro actuar el que se halla en la base del juego lingüistico" (13).

Y más adelante: "La pintura-del-mundo en su estadio 'practico' pre-proposicional se denomina también una forma de vida" (13).

Dreyfus, basándose en la tradición fenomenológica y particularmente en Maurice Merlau-Ponty, ha desarrollado en detalle una crítica radical del cognitivismo. Polemizando como Winograd y otros estudiosos de la inteligencia artificial, sostiene que los fenómenos cognitivos cotidianos no pueden nunca llegar a entenderse si se los considera como implicando un conjunto de contenidos o representaciones sobre los cuales la mente opera siguiendo reglas contextualmente libres. Por el contrario, el individuo está siempre dentro de una situación que "está determinada por nuestros cambiantes estados de ánimo, por nuestros proyectos y preocupaciones corrientes, por nuestras auto-interpretaciones de largo alcance y probablemente también por nuestras habilidades sensorio-motrices para enfrentarnos con objetos y personas sin habernos representado jamás nuestro cuerpo como un objeto, nuestra cultura como un conjunto de creencias y nuestras proposiciones como reglas de situaciones. Todas estas capacidades distintivamente humanas proveen una 'riqueza' o 'espesor' a nuestro modo de ser-en-el-mundo que puede jugar un rol esencial en la 'situacionalidad', la cual subyace, a sua vez, a toda conduct inteligente" (14).

(13) - Wittgenstein, L. (1969) On Certainty (Edited by G. E. M. Anscombe and G. H. von Wright). London, Harper \& Row, 204.

(14) - Dreyfus, J. Op. cit. 
Esta concepción se distingue de todas las variantes del cognitivismo, incluso de aquellas que conciben al conocimiento como estrechamente ligado a la acción y de las que, como en el caso de Walter Weimer (15), toman a las habilidades motoras como caso paradigmático. Ni Weimer ni John Bransford, ni ninguno de los teóricos cognitivos que, como el mismo Neisser, han buscado desarrollar una psicología cognitiva más "realista" que la clásica, han aparentemente renegado del postulado autonomista. A lo sumo, como en el caso de Weimer, en lugar de mirar al SCH desde "arriba", desde el pensamiento abstracto, lo miran desde "abajo", desde las habilidades motoras (motor skills). Pero no por ello dejan de suponer la existencia de un área cognitiva funcionalmente autónoma. Dreyfus, en cambio, no se limita a tomar el conocimiento exoceptual como central y de este modo invertir la perspectiva del SCH sino que, además, parece querer disolver o trascender la dicotomía entre lo cognitivo y lo no cognitivo. Su enfoque toma como punto de partida un ser humano que, por su condición misma de ser humano, tiene un cuerpo $\mathrm{y}$, además, está inevitablemente en un mundo de significados culturalmente originados y embebidos en prácticas sociales compartidas. Al igual que Wittgenstein, el conocimiento aparece así como imposible de disociar en última instancia de una forma de vida. Postular un "conocedor" dotado de un universo de representaciones y reglas autónomo es, según esta postura, completamente alejado de la realidad.

\section{Una alternativa: El modelo centro periferia}

El enfoque del SCH que acabamos de esbozar tiene el mérito, en oposición al modelo autonomista del cognitivismo, de destacar el hecho real de la inserción profunda del SCH en un cuerpo y en una cultura. Pero surge una duda: en su crítica del cognitivismo ino habrá ido demasiado lejos? Mas precisamente ¿es aceptable el rechazo total del supuesto 1, el supuesto de la autonomía funcional del SCH? Mi conjetura es que no. Para respaldar esta conjetura, adoptaré y esbozaré brevemente en lo que sigue un modelo centro-periferia del SCH de acuerdo con el cual existen ciertas áreas del sistema en que la suposición de autonomía funcional parece, al menos parcialmente, bastante adecuada.

Volvamos al tema de los esquemas. Tal vez el argumento más convincente en favor de la conservación de este constructo - tan persistente en la tradición cognitiva - es que sin él la influencia del

(15) - Cf. Weimer, W. B. (1977), A Conceptual Framework for Cognitive Psychology: Motor Theories of the Mind. En R. Shaw y J. Bransford (Eds.) Perceiving, Acting and Knowing: Toward an Ecological Psychology. 
pasado sobre el presente psicológico se vuelve difícil de explicar. Como dijera F. C. Bartlett: "La determinación mediante esquemas es la más tundamental de todas las maneras en que podemos ser influidos por las reacciones y experiencias que ocurrieron alguna vez en el pasado"'(16). Los esquemas, como lo hacen claro Piaget, Neisser y otros son pautas relativamente estables que se hallan en interacción con la experiencia: determinan la experiencia y son determinadas por ella. Están formados, en grado variable, por componentes simbólicos, representacionales, conductales, etc., dependiendo el peso de cada uno del nivel del SCH al que corresponden. En los niveles más inferiores, los aspectos representacionales y simbólicos prácticamente desaparecen en tanto que predominan en los niveles superiores.

Parece legítimo admitir la existencia en todo $\mathrm{SCH}$ desarollado de una multitud de esquemas (o estructuras, o marcos, o como quiera llamárseles) de distinto tipo. Un niño presenta relativamente pocos esquemas; como es sabido, en una etapa temprana ni siquiera el esquema del cuerpo propio se halla formado. Pero a medida que el niño crece no sólo aumenta el número de esquemas sino que también aumenta el número de conexiones entre los esquemas. Dicho de otro modo, el $\mathrm{SCH}$ no sólo crece en cantidad de elementos sino también en organización (17).

Los modos de vinculación entre los esquemas son de muy diverso tipo y suelen intervenir conjuntamente en la formación de conjuntos asociados de esquemas, que denominaremos subsistemas cognitivos. Tenemos así vínculos de tipo más objetivo, es decir, basados en características intersubjetivamente apreciables tales como las relaciones espaciales, temporales y lógicas, y vínculos de tipo más subjetivo, más estrechamente ligados con modos privados de construcción de la realidad. Algunos autores, por ejemplo Kevin Lynch en The Image of the City o el mismo Neisser en sua adaptación de la noción tolmaniana de mapa cognitivo, parecen olvidarse de que en la imagen mental de la realidad existe por lo general una superposición entre ambos tipos de vínculos y que no obran solamente vínculos de tipo objetivo. Sin embargo, debe reconocerse que el desarrollo del $\mathrm{SCH}$ también implica normalmente una disminución en el grado de superposición entre ambos tipos de vínculos.

Un ejemplo de subsistema cognitivo podría ser, tomando el sistema cognitivo de un niño, el formado por los esquemas de su madre, su padre, sus hermanos, la casa en que vive; o el formado por los

(16) - Bartlett, F. C. (1932) Remembering. Cambridge University Press, p. 201.

(17) - Cf. Witkin, H. A. et al. (1974) Phychological Differenciation. N. York, J. Wiley. 
mos dividir esa zona en esquemas y subsistemas claramente diferenciados entre sí. Esto es así ante todo porque el esquema central, el esquema del yo, se puede suponer como íntimamente penetrado por otros esquemas, por los esquemas de "otros significativos", el esquema del cuerpo propio, los esquemas correspondientes a creencias básicas de la cultura y hasta esquemas correspondientes a objetos materiales cargados con una alta significación subjetiva. $\mathrm{Y}$ así como el esquema del yo no es claramente diferenciable de otros esquemas, otros esquemas centrales, aunque en menor proporción, tampoco lo son. Hay aquí una fuerte superposición entre las distintas zonas de significación, una mezcla compleja de resonancias, como señalara James ó como ocurre en el modelo holográfico de Carl Pribran. El modo de pensamiento correspondiente a esta zona central del SCH es, correspondientemente, alejado de la lógica verbal, unas veces disperso y centrífugo y otras veces holístico, capaz de unir elementos que el pensamiento verbal difícilmente asocia. En cambio, a medida que nos alejamos del centro en dirección a la periferia, el grado de solapamiento entre los esquemas disminuye y el modo de pensamiento tiende a ser lógico-verbal, por pasos, analítico, cartesiano.

Otra dimensión fuertemente asociada con la dimensión centroperiferia (y por lo tanto con las dimensiones relacionadas, el número y tipo de conexiones entre esquemas) es la dimensión afectiva. Esta asociación se revela especialmente a través de la reacción emotiva que suele desencadenar la presentación de estímulos incompatibles con elementos del sistema cognitivo. Esto ha llevado a postular la existencia de mecanismos de defensa cognitivos. Así, por ejemplo, de acuerdo con Rokeach, toda información es escrutada para determinar si es compatible con el sistema. Si lo es, se admite su incorporación al sistema pero si no lo es se produce una reacción emotiva cuya magnitud es inversamente proporcional con el grado de centralidad del esquema o subsistema. La estrategia cognitiva subsiguiente a la recepción de una información incompatible con creencias centrales es o bien un rechazo de la información, o bien una distorsión de la misma de modo de eliminar sus aspectos incompatibles o bien un registro fiel de la información pero conservándola aislada del resto del sistema cognitivo (en la periferia). Existen en este punto sin embargo, como lo señala Rokeach, considerables diferencias individuales que van desde, en un extremo, individuos cuyos sistemas cognitivos son impenetrables para materiales disonantes más allá de las zonas estrictamente periféricas hasta, en el otro extremo, individuos cuyos sistemas cogritivos carecen de mecanismos de defensa rígidos y pueden llegar a admitir cambios en sus sistemas centrales.

Independientemente de la plausibilidad de modelos como el de Rokeach, en parte comprometidos con un enfoque de procesamiento 
de la información, y por lo tanto pasibles hasta cierto punto de las críticas que hicimos en la sección anterior a este enfoque, la relación entre cognición y afectividad es hoy ampliamente reconocida en psicología. Pero lo que generalmente no se toma debidamente en cuenta es que no todos los contenidos cognitivos están afectivamente cargados por igual. De acuerdo con la descripción anterior, las cogniciones podrían ordenarse según una línea de afectividad decreciente que, partiendo de la región central del $\mathrm{SCH}$, de afectividad fuerte y masiva, avanza hacia las zonas periféricas, de afectividad débil y difusa. Al principio del trayecto, en plena región central, es tal vez equivocado hablar de cogniciones y afectos como si fueran entidades separadas que entran en relación. Podríamos decir, que la región central está constituida por "afecto-esquemas", con predominio del componente afectivo sobre el cognitivo y que a medida que nos alejamos de ella, la relación se invierte hasta alcanzarse un predominio del componente cognitivo. Dicho de otro modo, el SCH está, en su región central, fusionado con el sistema afectivo-motivacional y se va separando del mismo a medida que se avanza hacia las regiones más externas.

El modelo centro-periferia del $\mathrm{SCH}$ que venimos describiendo podría tal vez hacerse algo menos abstracto, acercarse más a la experiencia subjetiva, si lo viéramos a la luz de una sugestiva teoría elaborada por William James (20) y retomada varios años después por Alfred Schutz (21). De acuerdo con esta teoría deberían distinguirse, dentro del conjunto de la experiencia individual, diferentes "submundos", como decía James o "regiones finitas de significado", como prefería decir Schutz. Cada uno de estos submundos se caracterizaría por un peculiar conjunto de reglas constitutivas y por una peculiar definición de realidad. (En cuanto a la primera característica, los micro-mundos de Minsky serían la tradución de la misma idea al lenguaje de la inteligencia artificial). Schutz agregó a esto la afirmación de que el paso de una región finita de significado a otra implica una especia de 'shock' y de salto. "Son tantas las clases diferentes de experiencias de "shock" como diferentes regiones de significado a las que puedo conceder el acento de realidad. Algunos ejemplos son: el 'shock' de caer dormido así como el salto al mundo de los sueños; la transformación interna que padecemos cuando al levantarse el telón del teatro se marca la transición al mundo de la escena" (22).

(20) - James, W. (1950) Principles of Psychology, vol. 2. New York. Dover Publications, cap. 21 "The Perception of Reality".

(21) - Schutz, A. (1945) On Multiple Realities, en A. Schutz, Collected Papers: I. The Problem of Social Reality. The Hague, Martinus Nijhoff.

(22) - Schutz, A. Op. cit., p. 231. 
Schutz se esfuerza por determinar la actitud característica de cada región finita de significado. Con respecto, por ejemplo, a la actitud del científico teórico señala do siguiente: "El salto al ámbito del pensamiento teórico supone la resolución del individuo de suspender su punto de vista subjetivo. $Y$ este hecho muestra por sí solo que no es el sí-mismo indiviso el que "actúa" en el ámbito del pensamiento teórico, sino un sí-mismo parcial, alguien que asume un rol, un "Mí", o sea, el teórico. Este sí-mismo parcial carece de todas las experiencias 'esencialmente actuales' y todas las experiencias vinculadas con su proprio cuerpo, sus movimientos y sus límites" (23).

En relación con la realidad cotidiana, Schutz, en coincidencia con James, la considera como siendo una región de significado más, con sus peculiares reglas constitutivas y su peculiar definición de realidad. Está dotada, sin embargo, de un status privilegiado; es la primera entre las realidades, por así decirlo, ya que suele triunfar en la confrontación con ellas. Llamamos a un modelo del SCH como el de Schutz (semejante en lo esencial al de Minsky) "modelo autonomista" ya que divide a este sistema en regiones finitas de significado mutuamente autónomas. Si tuviéramos que representarlo mediante un esquema, diríamos que, en un instante $t_{1}$ tendrá la seguiente forma

Fig. 2: Modelo Autonomista del SCH. La zona rayada representa la región finita de significado $R_{1}$ en que se encuentra la conciencia en el instante $t_{i}$.

El modelo James-Schutz es discutible en muchos aspectos. En primer lugar, es discutible la consideración de la realidad cotidiana como un "mundo" de algún modo paralelo o comparable a los demás. Nos parece muy relevante aquí la observación de Erving Goffman cuando sostiene que la teoría James-Schutz no ha tenido "mucho éxito en describir las reglas constitutivas de la actividad cotidiana" Se propone - agrega Goffman — una similaridad estructural entre

(23) - Schutz, A. Op. cit., p. 250. 
la vida cotidiana. y los diversos 'mundos' de creencia-actividad pero no se ve cómo esta relación puede modificar nuestra concepción de la vida cotidiana" (24).

En segundo lugar, el carácter cerrado y autónomo que el modelo James-Schutz atribuye a los diferentes mundos, su falta de interacción mutua, parece una real exageración. Así, por ejemplo, lo es, sin duda, la afirmación de Schutz de que un teórico pierde al estar en el mundo de la teoría, "todas las experiencias vinculadas con su propio cuerpo, sus movimientos y sus límites" La generalización hecha por Schutz de las nociones de shock y de salto a todo paso de un "mundo" a otro parece ser simplemente una dudosa consecuencia de esa exagerada distinción entre distintos "mundos" Sin embargo, y a pesar de estas críticas, debería reconocerse que el modelo JamesSchutz apunta a una característica central del SCH, a saber, su diferenciación en subsistemas. Retomando el modelo centro-periferia, podríamos entender tanto las limitaciones como los hallazgos del modelo James-Schutz. Por una parte, al caracterizar a la zona central como constituida por esquemas interpenetrados y con límites difusos, que se pierden progresivamente en un horizonte ilimitado, el modelo centro-periferia muestra la impossibilidad de considerar a las experiencias en que la zona central está en juego, o al menos en estado de alerta, como un mundo determinado por un conjunto de reglas. Esto requeriría, como vimos en la sección anterior, la existencia de áreas bien delimitadas de significado, áreas que no existen en la zona central del SCH, como el mismo James mostrara en otro lugar. Por otra parte, al caracterizar a las zonas periféricas como formadas por "subsistemas cognitivos diferenciados", el modelo señala la aplicabilidad allí de la teoría James-Schutz. No es posible olvidar, sin embargo, que ni siquiera aquí la autonomía entre los subsistemas cognitivos es tan amplia como lo requiere la teoría James-Schutz (salvo, tal vez, en casos de disociación patológica). Pero no caben dudas de que cierta diferenciación experiencial en la línea señalada por JamesSchutz corresponde a la realidad.

\section{Observaciones finales}

$\mathrm{Ni}$ el cognitivismo ni el anticognitivismo parecen ser, pues, enfoques enteramente satisfactorios de los procesos psicológicos implicados en la generación, aplicación y cambio del conocimiento humano. Parecería necesaria una tercera alternativa, capaz de incorporar los insights de ambos pero que no sea una mera combinación sino una alternativa creadora que pueda romper el actual impasse. Esta alter-

(24) - Goffman, E. (1974) Frame Analysis: An Essay on the Organization of Experience. New York, Harper \& Row, p. 6. 
nativa debería fundarse en el reconocimiento de que una peculiar mezcla de autonomía y compromiso respecto de la zona central del SCH caracteriza a cada uno de los subsistemas cognitivos diferenciados. Sin la posibilidad de tomar distancia en relación con las zonas más comprometidas con la subjetividad ningún conocimiento exacto y objetivo del mundo sería posible pero sin la posibilidad de sumergirse en esa zona del $\mathrm{SCH}$ el conocimiento probablemente no podría dar verdaderos saltos creadores y emprender caminos radicalmente nuevos. Las razones para ello son complejas y las hemos bosquejado en otros lugares (25). Podríamos resumirlas brevemente aquí diciendo que concebimos a la zona central del $\mathrm{SCH}$ como una especie de reservorio que contiene, por una parte, reflejos distorsionados debilitados en su estructura lógica - de las relaciones cristalizadas en los subsistemas cognitivos diferenciados y, por otra parte, incipientes posibilidades de relaciones no desarrolladas. Algunas de estas posibilidades de relación pueden en algún momento empezar a activarse y llegar a emerger hasta convertirse, a su vez, en subsistemas cognitivos diferenciados.

Habría que admitir, pues, tanto la existencia de procesos cognitivos relativamente autónomos, en que la conciencia "habita" usando la terminología de Polanyi, en uno de los subsistemas diferenciados, como la existencia de procesos de conexión entre esos subsistemas y la zona central del SCH, procesos que a veces tienen el poder de desestructurar aquellos subsistemas y producir otros en su lugar. Un mo:lelo que pueda dar cuenta simultáneamente de la atracción que ejercen ambos polos del SCH - la exploración en la obscuridad de la zona central y el juego dentro de subsistemas periféricos relativamente claros y articulados - debería ser en principio más satisfactorio que los modelos corrientes que solo prestan atención a una de las caras janusianas del proceso humano de logro del conocimiento.

Fundación Bariloche, Argentina.

(25) - Cf. Nudler, O. (1976) Epistemología, Psicología y Cambio Científico. Crítica. Revista Hispanoamericana de Filosofía, Vol. VIII, N. ${ }^{\circ} 24$; Notes for an Epistemology of Holism (1979). The United Nations University, Tokyo; Human Needs: A Sophisticated Holistic Approach (1980), em K. Lederer (ed.) Human Needs. A Contribution to the Current Debate, Oelgenschlager, Berlin. 